\title{
Including the Effect of a Future Test and Redesign in Reliability Calculations
}

\author{
Diane Villanueva, $\stackrel{*}{-}$ Raphael T. Haftka,, \pm and Bhavani V. Sankar $\stackrel{ \pm}{ \pm}$ \\ University of Florida, Gainesville, Florida 32611
}

DOI: $10.2514 / 1 . J 051150$

\begin{abstract}
It is common to test components after they are designed and redesign if necessary. The reduction of the uncertainty in the probability of failure that can occur after a test is usually not incorporated in reliability calculations at the design stage. This reduction in uncertainty is accomplished by additional knowledge provided by the test and by redesign when the test reveals that the component is unsafe or overly conservative. In this paper, a methodology is developed to estimate the effect of a single future thermal test followed by redesign and to model the effect of the resulting reduction of the uncertainty in the probability of failure. Using assumed distributions of computation and experimental errors and given redesign rules, possible outcomes of the future test and redesign through Monte Carlo sampling are obtained to determine what changes in probability of failure, design, and weight will occur. In addition, Bayesian updating is used to gain accurate estimates of the probability of failure after a test. These methods are demonstrated through a future thermal test on an integrated thermal protection system. Performing redesign following a single future test can reduce the probability of failure by orders of magnitude, on average, when the objective of the redesign is to restore original safety margins. Redesign for a given reduced probability of failure allows additional weight reduction.
\end{abstract}

\section{Nomenclature}

$C \quad=$ capacity

$D_{L} \quad=$ lower bound for deterministic redesign criterion

$D_{U} \quad=$ upper bound for deterministic redesign criterion

$d \quad=$ design variable

$d_{s} \quad=$ foam thickness, $\mathrm{m}$

$e_{c} \quad=$ computational error

$e_{\text {extrap }}=$ extrapolation error

$e_{x}=$ experimental error

$f(T) \quad=$ probability distribution of the temperature

$g \quad=$ limit state function

$I=$ indicator function

$l_{\text {test }}(T)=$ likelihood function of obtaining the test-article temperature

$M \quad=$ number of samples of the capacity

$m \quad=$ mass per unit area, $\mathrm{kg} / \mathrm{m}^{2}$

$N=$ number of samples of the response

$P_{L} \quad=$ lower bound for probabilistic redesign criterion

$P_{U} \quad=\quad$ upper bound for probabilistic redesign criterion

$p_{f \text {,analyst }}=$ analyst-estimated probability of failure, $\%$

$p_{f, \text { target }}=$ probabilistic redesign probability of failure target, $\%$

$p_{f, \text { true }}=$ true probability of failure, $\%$

$R=$ response

$r \quad=$ random variable

$T=$ temperature, $\mathrm{K}$

$\Delta d_{\lim } \quad=$ limit of distance between test design and other design

Presented at the 12th AIAA Non-Deterministic Approaches Conference, Orlando, FL, 12-15 April 2010; received 30 December 2010; revision received 20 March 2011; accepted for publication 8 May 2011. Copyright (@) 2011 by Diane Villanueva, Raphael T. Haftka, and Bhavani V. Sankar. Published by the American Institute of Aeronautics and Astronautics, Inc., with permission. Copies of this paper may be made for personal or internal use, on condition that the copier pay the $\$ 10.00$ per-copy fee to the Copyright Clearance Center, Inc., 222 Rosewood Drive, Danvers, MA 01923; include the code 0001-1452/11 and $\$ 10.00$ in correspondence with the CCC.

*Graduate Research Assistant, Department of Mechanical and Aerospace Engineering. Student Member AIAA.

Distinguished Professor, Department of Mechanical and Aerospace Engineering. Fellow AIAA.

*Ebaugh Professor and University of Florida Research Foundation Professor, Department of Mechanical and Aerospace Engineering. Associate Fellow AIAA.

\section{Subscripts}

$\begin{array}{ll}\text { allow } & =\text { allowable } \\ \text { Bayes } & =\text { value obtained from Bayesian updating } \\ \text { calc } & =\text { calculated } \\ \text { corr } & =\text { corrected } \\ \text { ini } & =\text { initial } \\ \text { inp } & =\text { input } \\ \text { meas } & =\text { measured } \\ \text { test } & =\text { test article } \\ \text { true } & =\text { true } \\ P \text { true } & =\text { possible true }\end{array}$

Superscript

upd $\quad=$ updated

\section{Introduction}

I N RELIABILITY-BASED design optimization, uncertainties are considered when calculating the reliability of the structure. In the design process, uncertainty is often compensated for with safety factors and knockdown factors. However, after design, it is customary for the component to undergo various uncertainty-reduction measures (URMs). Examples of URMs in the aerospace field include thermal and structural testing, inspection, health monitoring, maintenance, and improved analysis and failure modeling. Since most components undergo these URMs, it would be beneficial to include their effects in the design process.

In recent years, there has been a movement to quantify the effect of URMs on the safety of the product over its life cycle. Much work has been completed in the areas of inspection and maintenance for structures under fatigue loading. Fujimoto et al. [1], Toyoda-Makino [2], and Garbatov and Soares [3] developed methods to optimize inspection schedules for a given structural design to maintain a specific level of reliability. Even further, Kale et al. [4,5] explored how simultaneous design of the structure and inspection schedule allows the trading of cost of additional structural weight against inspection cost of stiffened panels affected by fatigue crack growth.

However, there are few studies that have incorporated the effects of future tests followed by possible redesign on the design of a structure. Studies by Acar et al. [6,7] investigated the effects of future tests and redesign on the final distribution of failure stress and structural design with varying numbers of tests at the coupon, element, and 

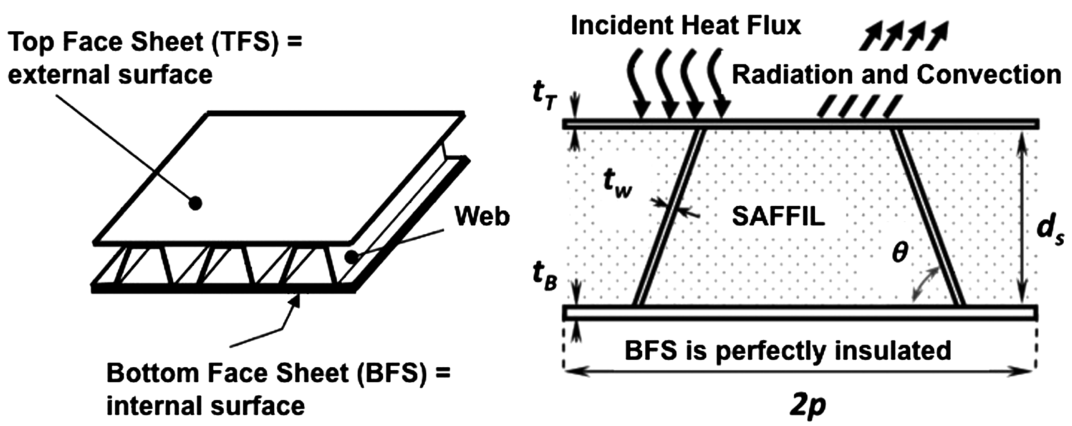

Fig. 1 Corrugated-core sandwich-panel ITPS concept.

certification levels. Such studies showed that these tests with possible redesign can greatly reduce the probability of failure, and they estimated the required structural weight to achieve the same reduction without tests.

In this study, we examine the effect of a single future thermal test followed by possible redesign on the reliability and weight of an integrated thermal protection system (ITPS). An integrated thermal protection system protects space vehicles from the severe aerodynamic heating experienced upon atmospheric reentry while also providing some structural load bearing benefit. The thermal test considered in this study measures the maximum temperature of the bottom face sheet, which is critical due to its proximity to the underlying vehicle structure. A design is considered to have failed thermally if it exceeds the maximum allowable temperature.

In previous work on the optimization of the ITPS, Villanueva et al. [8] used probability of failure calculations that considered only the variability in geometric and material parameters and error due to shortcomings in the analytical model. Expanding on those studies, we include the information gained from a test in a temperature estimate, the reduction in uncertainty resulting from the test, and the ability of the test to guide redesign for dangerous or overly conservative designs. Thereby, the objectives of this paper are as follows:

1) Present a methodology to both predict and include the effect of a future redesign following a test during the design stage.

2) Illustrate the ability of a test in combination with redesign to reduce the probability of failure even when a test shows that the design is computationally unconservative.

3) Examine the overall changes in mass resulting from redesign based on the future test.

A description of the ITPS is presented in Sec. II. Next, the uncertainty model and probability of failure calculations are described in Sec. III. Section IV continues with the methodology to calibrate the computational model based on a test and includes redesign based on the test. The method to simulate future tests is summarized in Sec. V. Section VI presents an illustrative example that details the effect of including the test and redesign in probability of failure calculations. The paper then concludes with a summary and ideas for future work in Sec. VII.

\section{ITPS Description}

Figure 1 shows the ITPS panel being studied, which is a corrugated-core sandwich-panel concept. The design consists of a top face sheet and webs made of titanium alloy (Ti-6Al-4V) and a bottom face sheet made of beryllium. Saffil ${ }^{\circledR}$ foam is used as insulation between the webs. The relevant geometric variables of the ITPS design are also shown on the unit cell in Fig. 1. These variables are the top face thickness $t_{T}$, bottom face thickness $t_{B}$, thickness of the foam $d_{S}$, web thickness $t_{w}$, corrugation angle $\theta$, and length of unit cell $2 p$.

Thermal analysis of the ITPS is done using 1-D heat transfer equations on a model of the unit cell. The heat flux incident on the top face sheet of the panel is highly dependent on the vehicle shape as well as the vehicle's trajectory. As in previous studies by Bapanapalli [9], incident heat flux on a Space Shuttle-like vehicle was used. A large portion of the heat is radiated out to the ambient by the top face sheet, and the remaining portion is conducted into the ITPS. We consider the worst-case scenario, where the bottom face sheet cannot dissipate heat, by assuming that the bottom face sheet is perfectly insulated. Also, there is no lateral heat flow out of the unit cell, so that heat flux on the unit cell is absorbed by that unit cell only. For a more in-depth description of the model and boundary conditions, the reader is referred to the Bapanapalli reference.

The maximum temperature of the bottom face sheet of the ITPS panel is calculated using the quadratic response surface developed by Villanueva et al. [8] by a process similar to that of Gogu et al. [10], using the MATLAB toolbox developed by Viana.. It is a function of the previously described geometric variables and the density, thermal conductivity, and specific heat of titanium alloy, beryllium, and Saffil ${ }^{\circledR}$ foam. The mass per unit area $m$ of the ITPS is calculated using Eq. (1), where $\rho_{T}, \rho_{B}$, and $\rho_{w}$ are the densities of the materials that make up the top face sheet, bottom face sheet, and web, respectively:

$$
m=\rho_{T} t_{T}+\rho_{B} t_{B}+\frac{\rho_{w} t_{w} d_{S}}{p \sin \theta}
$$

An experiment that finds the bottom face sheet temperature of a small ITPS panel is usually conducted in a vacuum chamber with heat applied to the top face sheet by heat lamps. The sides of the panel are typically surrounded by some kind of insulation to prevent lateral heat loss. The temperature of the bottom face sheet is found with thermocouples embedded into or in contact with the lower surface of the bottom face sheet.

\section{Uncertainty Modeling}

\section{A. Classification of Uncertainties}

Oberkampf et al. [11] provided an analysis of different sources of uncertainty in engineering modeling and simulation, which was simplified by Acar et al. [6]. We use a similar classification to categorize types of uncertainty as errors (uncertainties that apply equally to every ITPS) or variability (uncertainties that vary in each individual ITPS). We further describe errors as mostly epistemic and variability as aleatory. It is important to distinguish between types of uncertainty because a specific uncertainty-reduction measure may target either error or variability. Tests reduce errors by allowing us to calibrate analytical models. For example, testing can be done to reduce the uncertainty in failure predictions due to high stresses. Variability can be reduced by lowering tolerances in manufacturing. Variability is modeled as random uncertainties that can be modeled probabilistically. In contrast, errors are fixed for a given ITPS and are largely unknown, but here they are modeled probabilistically as well.

Variability in material properties and construction of the ITPS leads to variability in the ITPS thermal response. More specifically, we will have variability in the calculated temperature, due to the input variabilities. We simulate this process with a Monte Carlo simulation that generates values of the random variables $r$ based on an estimated distribution and calculates the bottom face sheet temperature $T_{\text {calc }}$ for

\footnotetext{
${ }^{\S}$ Data available online at http://sites.google.com/site/felipeacviana/.
} 
each, generating the probability distribution function. The calculated temperature distribution that reflects the random variability is denoted $f_{\text {calc }}(T)$. In estimating the probability of failure, we also need to account for the modeling or computational error. We denote this computational error by $e_{c}$, where $e_{c}$ is modeled as a uniformly distributed random variable within confidence limits the in the computational model as defined by the analyst. Unlike the variability, the error has a single value, and the uncertainty is due to our lack of knowledge.

For a given design given by $d$ and $r$, the possible true temperature $T_{P \text { true }}$ can be found by Eq. (2) in terms of possible computational errors $e_{c}$. The sign in front of $\bar{e}_{c}$ is negative, so a positive error implies a conservative calculation, meaning that it overestimates the temperature:

$$
T_{P \text { true }}\left(d, r, e_{c}\right)=T_{\text {calc }}(d, r)\left(1-e_{c}\right)
$$

Since the analyst does not know $e_{c}$ and it is modeled as a random variable, we can form a distribution of the possible true temperature, denoted as $f_{P \text { true }}(T)$. To illustrate the difference between the true distribution of the temperature $f_{\text {true }}(T)$ and possible true distribution $f_{P \text { true }}(T)$, let us consider a simple example where the calculated temperature of the nominal design is 1 , the true temperature is 1.05 , and the computational error is uniformly distributed in the range $[-0.1,0.1]$. The possible true temperature without variability are uniformly distributed in the range $[0.9,1.1]$ by Eq. (2). Now, let us consider an additional variability in the temperature due to manufacturing tolerances in the range $[-0.02,0.01]$, such that $T_{\text {calc }}(d, r)$ is uniformly distributed in the range [0.98, 1.01]. Finally, the true temperature will vary from $[1.03,1.06]$ as $f_{\text {true }}(T)$, and the possible true temperature will vary from $[0.882,1.111]$ as $f_{P \text { true }}(T)$.

Figure 2 illustrates how we arrive at the distribution $f_{P \text { true }}(T)$. The input random variables have initial distributions, denoted as $f_{\text {inp }}(r)$, and these random variables, in combination with the design variables, lead to the distribution of the calculated temperature $f_{\text {calc }}(T)$. The random computational error is applied, leading to the distribution of the possible true temperature $f_{P \text { true }}(T)$, which has a wider distribution than $f_{\text {calc }}(T)$.

As previously noted, $e_{c}$ is modeled as a random variable not because it is random, but because its value is unknown to the analyst. To emphasize this point, the actual true temperature is known only when we know the actual value of $e_{c}$ as $e_{c \text {,true }}$ as illustrated in Eq. (3) below:

$$
T_{\text {true }}(d, r)=T_{\text {calc }}(d, r)\left(1-e_{c, \text { true }}\right)
$$

Again, these true values are unknown to the analyst. This distinction between true values and analyst-estimated, possible true values is important and will be a point of comparison throughout this paper.

Figure $\underline{3}$ shows an example of the probability distribution of the true temperature $f_{\text {true }}(T)$, as well as the probability density functions (PDFs) of $f_{\text {calc }}(T)$ and $f_{P \text { true }}(T)$. For this example, we modeled the variability in the material properties and variability in geometry with normal distributions, and we modeled the computational error with a uniform distribution. The plots of each PDF show the probability of exceeding the allowable temperature $T_{\text {allow }}$, represented by the area where the temperature exceeds the allowable.

We chose an illustration where the computational error is unconservative so the $f_{\text {calc }}(T)$ provides an underestimate of the

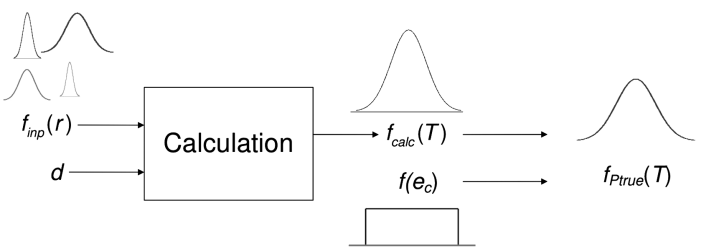

Fig. 2 Illustration of the variability of the input random variables, calculated value, computational error, and resulting distribution of possible true temperature. probability of failure given by $f_{\text {true }}(T)$. This computational error between the mean of $f_{\text {calc }}(T)$ and the mean of $f_{\text {true }}(T)$ is $e_{c \text {,true }}$. However, since we include $e_{c}$ as a random variable, we widened the distribution $f_{\text {calc }}(T)$, resulting in $f_{P \text { true }}(T)$. This provides a more conservative estimate of the probability that can compensate for the unconservative calculation. Of course, when the error in the calculation is conservative, this wide distribution will grossly overestimate the probability of failure.

\section{B. True Probability of Failure Calculation}

The true probability of failure of a design $d$ with random variables $r$ can be found when the true computational error is known. This is clearly a hypothetical situation because in reality the true computational error is not known by the analyst. Here, Monte Carlo simulation is used to calculate the true probability of failure. The limit state equation $g$ is formulated as the difference between a capacity $C$ and response $R$ as shown in Eq. (4):

$$
g=T_{\text {allow }}-T_{\text {true }}(d, r) \equiv C-R
$$

Since we consider failure to occur when the maximum bottom face sheet temperature exceeds the allowable temperature $T_{\text {allow }}$, the response is $T_{\text {true }}$ and the capacity is the allowable temperature. The true probability of failure $p_{f \text {,true }}$ is estimated with Eq. ()ㅡ:

$$
p_{f, \text { true }}=\frac{1}{N} \sum_{i=1}^{N} I\left[g\left(C_{i}, R_{i}\right) \leq 0\right]
$$

The indicator function $I$ equals 1 if the response exceeds the capacity, and it equals 0 for the opposite case. The number of samples is $N$.

\section{Analyst-Estimated Probability of Failure Calculation}

Since the true computational error is unknown, the true probability of failure is unknown as well. Because of this, the best estimate the analyst can obtain uses the calculated temperature $T_{\text {calc }}$ and the computational error through the possible true temperature of Eq. (2) to determine the estimated probability of failure with the limit state equation formulated as in Eq. (ㅁ):

$$
g=T_{\text {allow }}-T_{P \text { true }}\left(d, r, e_{c}\right) \equiv C-R
$$

Since the two types of uncertainty (computational errors and variability in material properties and geometry) in the response are independent, separable Monte Carlo sampling [12] can be used when evaluating the probability of failure. The limit state equation can be reformulated so that the computational error is on the capacity side, and all random variables associated with material properties and geometry lie on the response side:

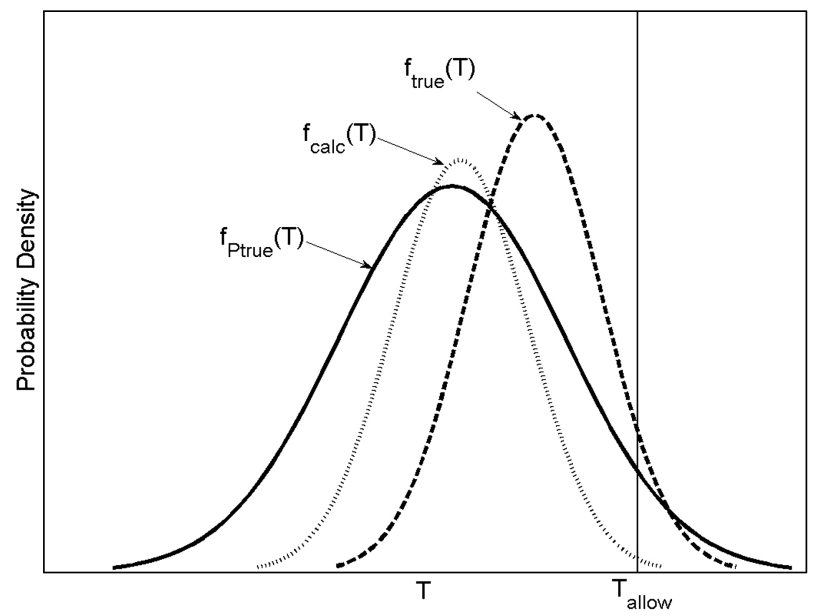

Fig. 3 Illustration with unconservative calculation of temperature when including the error in the estimate improves the estimate of the probability of failure. 


$$
g=\frac{T_{\text {allow }}}{1-e_{c}}-T_{\text {calc }}(d, r) \equiv C-R
$$

This analyst-estimated probability of failure $p_{f \text {,analyst }}$ can then be calculated with Eq. ( $\underline{8}$ ), where $M$ and $N$ are the number of capacity and response samples, respectively:

$$
p_{f, \text { analyst }}=\frac{1}{M N} \sum_{i=1}^{N} \sum_{j=1}^{M} I\left[g\left(C_{j}, R_{i}\right) \leq 0\right]
$$

\section{Including the Effect of a Calibration Test and Redesign}

We consider a test, performed for the purpose of validating and calibrating a model, for a selected design $d_{\text {test }}$ to determine the temperature of the test article $T_{\text {test }}$. We further assume that the test article is carefully measured for both $d_{\text {test }}$ and $r_{\text {test }}$ so that both are accurately known and that the errors in the computed temperatures due to uncertainty in the values of $d_{\text {test }}$ and $r_{\text {test }}$ are small compared with the measurement errors and can be neglected. If no errors are made in the measurements of $d_{\text {test }}, r_{\text {test }}$, and $T_{\text {test }}$, then the experimental result is actually the true temperature of the test article. We denote this error-free test temperature $T_{\text {test,true }}$ :

$$
T_{\text {test,true }}=T_{\text {true }}\left(d_{\text {test }}, r_{\text {test }}\right)
$$

However, there is unknown measurement error $e_{x}$, which we model as a random variable based on our estimate of the accuracy of the test. The measured temperature $T_{\text {meas }}$ then includes the experimental error $e_{x, \text { true }}$. The experimental error could also include a component due to the fact that $r_{\text {test }}$ is not perfectly known:

$$
T_{\text {meas }}=\frac{T_{\text {test,true }}}{1-e_{x, \text { true }}}
$$

Using the computational and experimental results, along with the corresponding error estimates for the test article, we are able to refine the calculated value and its error for any design described by the design variables $d$ and random variables $r$. In this way, the result of the single test can be used to calibrate calculations for other designs. We examine two methods, which take different approaches in using the test as calibration. The first approach introduces a simple correction factor based on the test result. The second uses the Bayesian method to update the uncertainty of the calculated value for $d_{\text {test }}$ based on the test result and then transfers this updated uncertainty to other calculations as the means of calibration.

\section{A. Correction-Factor Approach}

The correction factor approach is a fairly straightforward method of calibration. Assuming that the test result is more accurate than the calculated result for the test article, we scale $T_{\text {calc }}$ for any value of $d$ and $r$ by the ratio of the test result to the calculated result to obtain the corrected calculation $T_{\text {calc,corr }}$ :

$$
T_{\text {calc,corr }}=T_{\text {calc }}(d, r)\left(\frac{T_{\text {meas }}}{T_{\text {calc }}\left(d_{\text {test }}, r_{\text {test }}\right)}\right)
$$

\section{B. Bayesian-Updating Approach}

Before the test, we have an expectation of the test results based on the computational result of $d_{\text {test }}$ and $r_{\text {test }}$. We denote this distribution by $f_{\text {test }, P \text { true, }}^{\text {ini }}$ which can be viewed as the distribution of $f_{P \text { true }}(T)$ of the test article with fixed random variables $r_{\text {test }}$. Furthermore, it may be viewed as the possible true temperature distribution of the test article just before the test.

In the test, we measure a temperature $T_{\text {meas }}$. Because of experimental error $e_{x}$, the true test result $T_{\text {test,true }}$ is not equal to $T_{\text {meas }}$ (as seen in Eq. (10)). The possible true value of the test result is instead given as

$$
T_{\text {test }, P \text { true }}^{\text {meas }}=T_{\text {meas }}\left(1-e_{x}\right)
$$

where $T_{\text {test } \text { P } P \text { true }}^{\text {meas }}$ forms the distribution of possible true test results available from the measurements only. We thus have two distributions of possible true test results. One is based on the calculated value and the distribution of the calculation error, and the other is based on the measurement and the distribution of the measurement error.

The Bayesian approach combines these two distributions to obtain a narrower and more informative distribution. In this formulation, the probability distribution of the possible true temperature of the test article $f_{\text {test }, P \text { true }}(T)$ is updated as

$$
f_{\text {test }, P \text { true }}^{\text {upd }}(T)=\frac{l_{\text {test }}(T) f_{\text {test }, P \text { true }}^{\text {ini }}(T)}{\int_{-\infty}^{+\infty} l_{\text {test }}(T) f_{\text {test }, P \text { true }}^{\text {ini }}(T) \mathrm{d} T}
$$

where the likelihood function $l_{\text {test }}(T)$ is the conditional probability density of obtaining the test result $T_{\text {meas }}$ when the true temperature of the test article is $T$. That is, $l_{\text {test }}$ is the probability density of $T /(1-$ $e_{x}$ ) evaluated at $T=T_{\text {meas }}$.

The updated estimate $f_{\text {test }}^{\text {upd } P \text { true }}(T)$ is the distribution of the updated true possible test result $T_{\text {test }, P \text { true }}^{\text {upd }}$. This is used to find the distribution of the Bayesian estimate of the computational error $e_{\text {Bayes }}$ with Eq. (14):

$$
e_{\text {Bayes }}=1-\frac{T_{\text {test }, P \text { true }}^{\mathrm{upd}}}{T_{\text {calc }}\left(d_{\text {test }}, r_{\text {test }}\right)}
$$

We can then replace the possible true temperature given by Eq. (2) with a true temperature that uses the Bayesian estimate of the error:

$$
T_{P \text { true }}\left(d, r, e_{\text {Bayes }}\right)=T_{\text {calc }}(d, r)\left(1-e_{\text {Bayes }}\right)\left(1-e_{\text {extrap }}\right)
$$

The additional error $e_{\text {extrap }}$ is included to account for the error that occurs when applying this Bayesian estimate of the error to some design other than the test design. This extrapolation error is further described in Sec. IV.B.2.

Note that it is also possible to perform the Bayesian updating by reversing the roles of the two possible true test temperatures. That is, we could take the distribution based on the measurement error as the initial distribution and take the computed result as the additional information. However, in this case the likelihood function would require repeated simulations for different possible true temperatures, greatly increasing the computational cost.

\section{Illustrative Example of Calibration by the Bayesian Approach}

To illustrate how Bayesian updating is used to calibrate calculations based on a single future test, we consider a simple case where both the computational and experimental errors are uniformly distributed. To simplify the problem, we normalize all temperatures by the calculated temperature so that $T_{\text {calc }}\left(d_{\text {test }}, r_{\text {test }}\right)=1$. The error bound of the calculation is $\pm 10 \%$ and the error bound of the test is $\pm 7 \%$. The normalized test result is $T_{\text {meas }}=1.05$.

In this paper, we make the simplifying assumption that the likelihood function is about $T_{\text {meas }}$ rather than $T$. That is, we use conditional probability of obtaining the temperature $T$ given the measured temperature. This allows for a uniform value of the likelihood function where it is nonzero, which thereby results in a uniform distribution of the updated Bayesian estimate of the computational error since the distribution of $f_{\text {test, } P \text { true }}^{\text {upd }}$ will also be uniform. The effect of this approximation of the likelihood function is examined in Appendix A. The initial probability distribution $f_{\text {test }, P \text { true }}^{\text {ini }}(T)$ and the likelihood function $l_{\text {test }}$ are described by Eqs. (16) and (17), respectively:

$$
f_{\text {test }, P \text { true }}^{\text {ini }}(T)= \begin{cases}\frac{1}{0.2 T_{\text {calc }}\left(d_{\text {test }}, r_{\text {test }}\right)} & \text { if }\left|\frac{T}{T_{\text {calc }}\left(d_{\text {test }}, r_{\text {test }}\right)}-1\right| \leq 0.1 \\ 0 & \text { otherwise }\end{cases}
$$



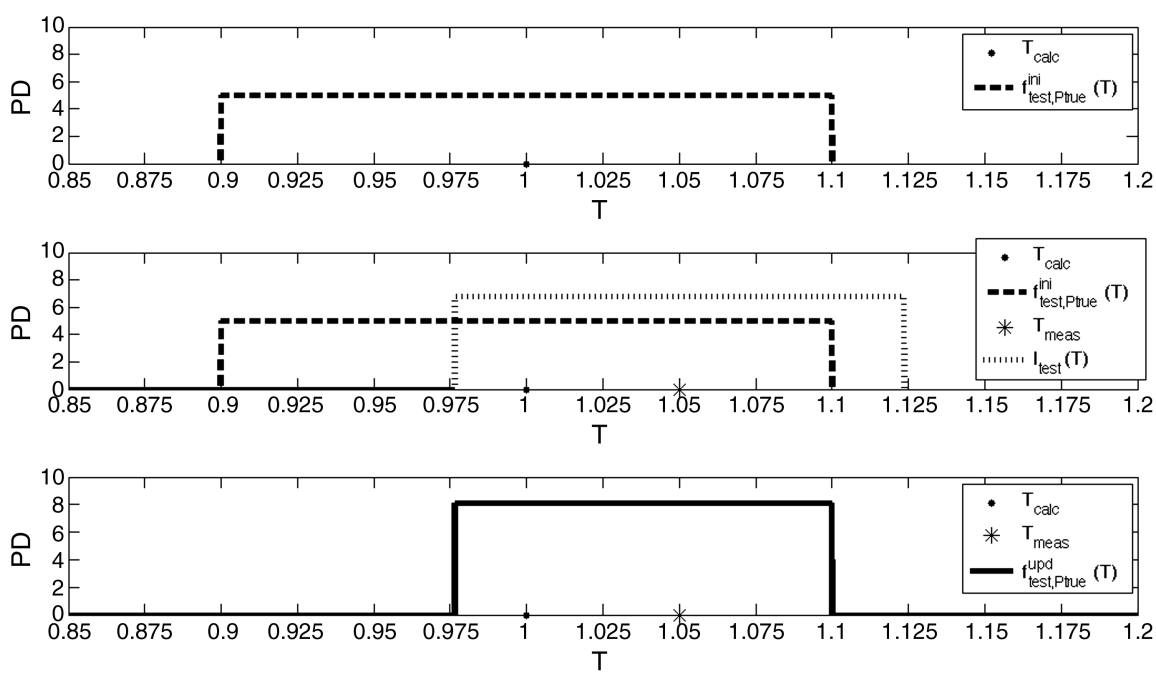

Fig. 4 Illustrative example of Bayesian updating showing the initial distribution (top), initial distribution and test (middle), and updated distribution (bottom).

$$
l_{\text {test }}(T)= \begin{cases}\frac{1}{0.14 T_{\text {meas }}} & \text { if }\left|\frac{T-T_{\text {meas }}}{T_{\text {meas }}}\right| \leq 0.07 \\ 0 & \text { otherwise }\end{cases}
$$

Since $T_{\text {calc }}\left(d_{\text {test }}\right)=1$ and the computation error bounds are $\pm 10 \%$, the initial distribution of the true temperature is $f_{\text {test }, P \text { true }}^{\text {ini }}(T)=5$ on the interval $(0.9,1.1)$ and zero elsewhere. This is shown in Fig. 4 . The test result of $T_{\text {meas }}=1.05$ results in a likelihood of $l_{\text {test }}=6.803$ on the interval $(0.9765,1.1235)$ and zero elsewhere. Equation (13) is used to find the updated $T_{\text {true }}$ distribution so that $f_{\text {test }, P \text { true }}^{\text {upd }}(T)=8.1$ on the interval $(0.9765,1.1)$ and zero elsewhere.

The updated distribution shows that the true temperature is somewhere on the interval $(0.9765,1.1)$. Using this temperature distribution along with the calculated value $T_{\text {calc }}\left(d_{\text {test }}\right)$, the updated error distribution $e_{\text {Bayes }}$ can be found. Through Eq. (14), we

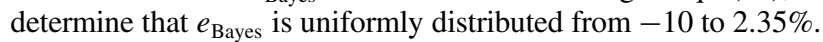

\section{Extrapolation Error in Calibration}

Figure 5 illustrates how the Bayesian approach is used to calibrate the calculations for other designs described by $d$. Here, we consider the case when the calculated temperature is linear in the design variable $d$, and there is no variability (random variables fixed at nominal values).

At design $d_{\text {test }}$, we have the same error scenario similar to that illustrated in Fig. 4. That is, we represent the calculated temperature at $d_{\text {test }}$ as a point on the black solid line, and the error bounds about this calculation by are represented by the black dotted lines. The star represents the experimentally measured temperature, and the error bars show the uncertainty in this temperature. By the Bayesian approach, we obtain a corrected test temperature as represented by

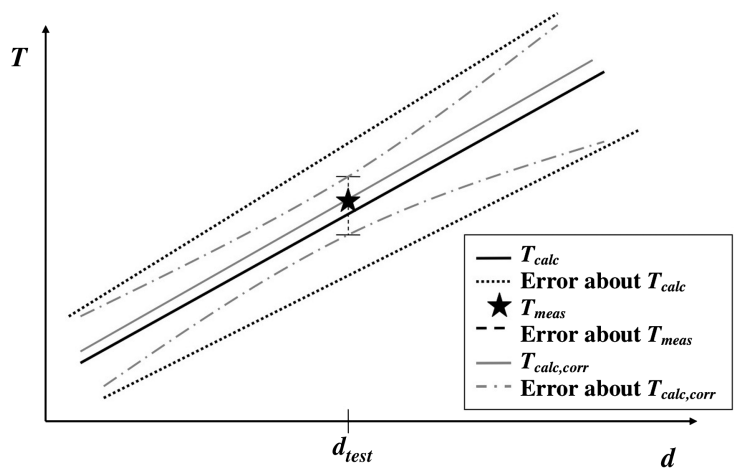

Fig. 5 Illustration of the calibration using Bayesian updating. the point on the gray line, as well as updated error bounds represented by the gray dash-dotted line.

However, this correction and updated error is most accurate at the test design. Therefore, we apply an additional error, the extrapolation error $e_{\text {extrap }}$, when calibrating designs other than $d_{\text {test }}$. Note that at $d_{\text {test }}$ the updated error bounds in Fig. 5 coincide with the error bounds of the test. As the design becomes increasingly different from $d_{\text {test }}$, the updated error bounds become wider.

The magnitude of $e_{\text {extrap }}$ is assumed to be proportional the distance between $d$ and $d_{\text {test }}$, such that

$$
e_{\text {extrap }}=\left(e_{\text {extrap }}\right)_{\max } \frac{\left\|d-d_{\text {test }}\right\|}{\Delta d_{\lim }}
$$

This defines the extrapolation error so that it is maximum when the distance between $d$ and $d_{\text {test }}$ is at limit of this distance $\Delta d_{\text {lim }}$ and zero at the test design. The extrapolation error is a measure of the variation of the errors in the model away from the test design. In this paper we assume that the magnitude of $e_{\text {extrap }}$ is linear with the distance between $d$ and $d_{\text {test }}$, which would be reasonable for small changes in the design. However, we examine the effect of this assumption in Appendix B, where we use a quadratic variation.

\section{Test-Corrected Probability of Failure Estimate}

The corrected probability of failure $p_{f \text {,analyst-corr }}$ after the test can be estimated by the analyst using the updated error obtained from the Bayesian approach. Separable Monte Carlo is used to calculate $p_{f, \text { analyst-corr }}$.

$$
\begin{gathered}
g=\frac{T_{\text {allow }}}{1-e_{\text {Bayes }}}-T_{\text {calc }}(d, r)\left(1-e_{\text {extrap }}\right) \equiv C-R \\
p_{f, \text { analyst-corr }}=\frac{1}{M N} \sum_{i=1}^{N} \sum_{j=1}^{M} I\left[g\left(C_{j}, R_{i}\right) \leq 0\right]
\end{gathered}
$$

\section{Redesign Based on the Test}

Two criteria for redesign are considered, each with different perspectives on the purpose of the redesign. The first criterion is based on the agreement between the measured and calculated values for the test article. The second criterion considers the probability of failure estimated by the analyst.

\section{Deterministic Redesign}

In deterministic redesign, redesign occurs when there is a significant difference between the experimentally measured temperature 


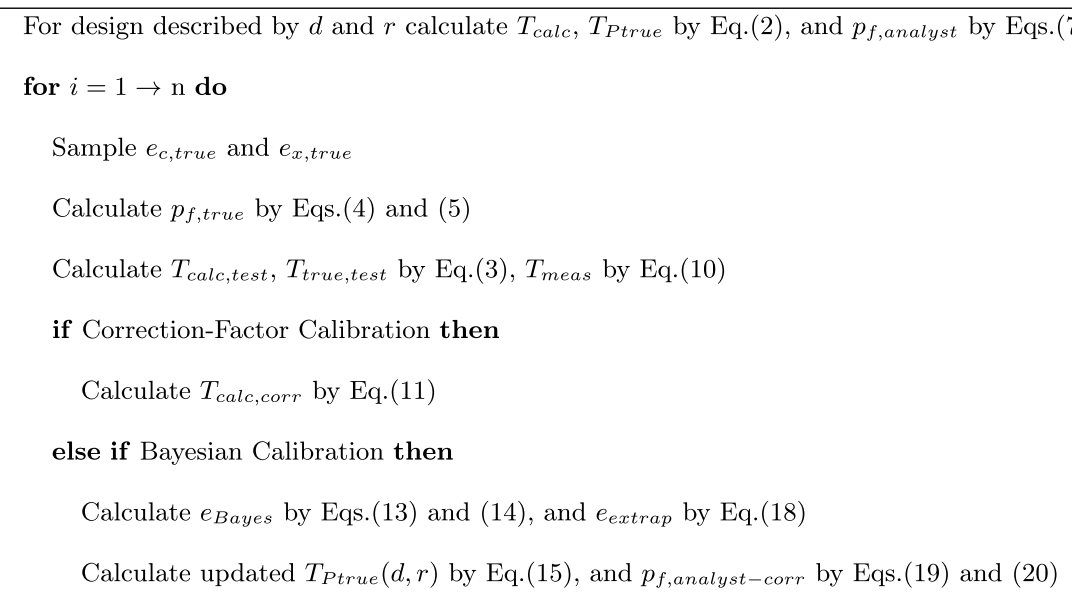

end if

if Deterministic Redesign then

if $\frac{T_{\text {meas }}}{T_{\text {calc }}\left(d_{\text {test }}, r_{\text {test }}\right)}<D_{L} \| \frac{T_{\text {meas }}}{T_{\text {calc }}\left(d_{\text {test }}, r_{\text {test }}\right)}>D_{U}$ then

Calculate $T_{\text {calc }}$ and $p_{f, t r u e}$ for redesign by Eqs.(4) and (5)

end if

else if Probabilistic Redesign then

if $p_{f, \text { analyst-corr }}>p_{f, \text { target }}+P_{U} \| p_{f, \text { analyst-corr }}<p_{f, \text { target }}-P_{L}$ then

Redesign for $p_{f, \text { analyst-corr }}=p_{f, \text { target }}$

Calculate $p_{f, t r u e}$ for redesign by Eqs.(4) and (5)

end if

end if

end for

Fig. 6 Procedure to simulate $n$ possible outcomes of a future test with redesign for a design described by $\boldsymbol{d}$ and $\boldsymbol{r}$.

$T_{\text {meas }}$ and the expected temperature given by the computational model. It is assumed that the temperature given by the computational model $\left(T_{\text {calc }}\right)$ is the desired value. Therefore, the component is redesigned to restore this original temperature.

The deterministic redesign criterion is implemented by imposing limits on the acceptable ratio of the measured temperature to the calculated temperature. Redesign occurs when $T_{\text {meas }} / T_{\text {calc }}\left(d_{\text {test }}, r_{\text {test }}\right)$ is less than the lower limit $D_{L}$ (conservative computational model) or exceeds the upper limit $D_{U}$ (unconservative computational model).

\section{Probabilistic Redesign}

In probabilistic redesign, the original structure is designed for a specified probability of failure, and redesign is also done to achieve a specified probability of failure. It is reasonable to select the target redesign probability $p_{f \text { target }}$ to be the same as that obtained with probabilistic design. The target redesign probability of failure can also be set to make the design safer after the test. Therefore, redesign occurs when the test-corrected probability of failure estimate, given by Eq. (20) is outside the limits of the acceptable range. The lower limit of this range is denoted as $P_{L}$, and the upper limit is denoted as $P_{U}$.

\section{Monte Carlo Simulations of a Future Test and Redesign}

Monte Carlo simulations are used to simulate the effect of a future test for a design described by design variables $d$ and random variables $r$ with the goal of simulating multiple possible outcomes of this test. To simulate a single outcome of the future test, we first obtain a single sample of the true computational and experimental errors.
Using the calculated value for the test design and the true computational error, we can obtain the true temperature by Eq. (3). Next, the experimentally measured temperature is found using Eq. (10). The choice can be made to calibrate by the correction factor approach or the Bayesian-updating approach, and, further, the choice of deterministic or probabilistic redesign can be made.

The true and corrected analyst-estimated probabilities of failure after the test can then be determined. At this point, the effect of only one possible outcome of the test has been examined. The major steps and equations involved in the simulation of a single outcome of the test are summarized in the pseudocode given in Fig. 6.1

To determine another possible outcome, the true computational and experimental errors are resampled and the process is repeated. Therefore, for $n$ possible outcomes of a future test, we sample $n$ pairs of the errors and true probabilities of failure, $n$ analyst-estimated probabilities of failure after the test, and up to $n$ updated designs. Note that there is a single initial design, but if $k$ of the $n$ cases are redesigned, we will end up with up to $k+1$ different designs.

\section{Illustrative Example}

In this example, we compare the probabilities of failure of an ITPS with the dimensions and material properties of probabilistic optimum found in [8]. In that study, the optimum was found with constraints on the maximum bottom face sheet temperature, buckling of the web, and maximum von Mises stress in the webs with the bottom face

\footnotetext{
"In the implementation of this algorithm, it is assumed that all analysts performing the test have the same value of $r_{\text {test }}$. Since each analyst accurately measured $r_{\text {test }}$, the effect of this assumption is likely to be negligible.
} 
Table 1 ITPS variables

\begin{tabular}{lccc}
\hline \hline Variable & Symbol & Nominal & CV, \% \\
\hline Web thickness & $t_{w}$ & $1.77 \mathrm{~mm}$ & 2.89 \\
Bottom face sheet & $t_{B}$ & $7.06 \mathrm{~mm}$ & 2.89 \\
Foam thickness & $d_{s}$ & $71.3 \mathrm{~mm}$ & 2.89 \\
Top face sheet thickness & $t_{T}$ & $1.2 \mathrm{~mm}$ & 2.89 \\
Half unit cell length & $p$ & $34.1 \mathrm{~mm}$ & 2.89 \\
Angle of corrugation & $\theta$ & $80^{\circ}$ & 2.89 \\
Density of titaniuma & $\rho_{\mathrm{Ti}}$ & $4429 \mathrm{~kg} / \mathrm{m}^{3}$ & 2.89 \\
Density of beryllium- & $\rho_{\mathrm{Be}}$ & $1850 \mathrm{~kg} / \mathrm{m}^{3}$ & 2.89 \\
Density of foam & $\rho_{S}$ & $24 \mathrm{~kg} / \mathrm{m}^{3}$ & 0 \\
Thermal conductivity of titanium & $k_{\mathrm{Ti}}$ & $7.6 \mathrm{~W} /(\mathrm{m} / \mathrm{K})$ & 2.89 \\
Thermal conductivity of beryllium & $k_{\mathrm{Be}}$ & $203 \mathrm{~W} /(\mathrm{m} / \mathrm{K})$ & 3.66 \\
Thermal conductivity of foam & $k_{S}$ & $0.105 \mathrm{~W} /(\mathrm{m} / \mathrm{K})$ & 2.89 \\
Specific heat of titanium & $c_{\mathrm{Ti}}$ & $564 \mathrm{~J} /(\mathrm{kg} / \mathrm{K})$ & 2.89 \\
Specific heat of beryllium & $c_{\mathrm{Be}}$ & $1875 \mathrm{~J} /(\mathrm{kg} / \mathrm{K})$ & 2.89 \\
Specific heat of foam & $c_{S}$ & $1120 \mathrm{~J} /(\mathrm{kg} / \mathrm{K})$ & 2.89 \\
\hline \hline
\end{tabular}

${ }^{\mathrm{a}}$ Top face and web material.

bBottom face material.

sheet, web thickness, and foam thickness as the design variables. The failure considered here is exceeding the allowable bottom face sheet temperature $T_{\text {allow }}$. All random variables are normally distributed with the mean and coefficient of variation (CV) shown in Table 1.

In this example, we consider uniform distributions of the errors, with the experimental error significantly smaller at $\pm 3 \%$ than the computational error at $\pm 10 \%$, as shown in Table 2 . The original estimated probability of failure is $0.121 \%$ and the nominal mass per unit area is $35.1 \mathrm{~kg} / \mathrm{m}^{2}$. Since the distributions of the errors are bounded, we remove the possibility of extreme differences between the calculated and experimentally measured values in the simulated future test. With these values of the errors, in the most extreme case, the temperatures differ by approximately $13 \%$, which occurs when the errors are sampled at opposing bounds of the distribution (e.g., $e_{c \text {,true }}=0.1$ and $e_{x \text {,true }}=-0.03$ ). If normal distributions of the errors were used, this difference can become infinite.

The extrapolation error $e_{\text {extrap }}$ is estimated to be $2 \%$ when $d$ is changed by $\pm 10 \%$ from $d_{\text {test }}$ and varies linearly with change in $d$ :

$$
e_{\text {extrap }}=0.02 \frac{\left\|d-d_{\text {test }}\right\|}{0.1\left\|d_{\text {test }}\right\|}
$$

It is possible to assume other relationships between of the extrapolation error and the distance of $d$ from $d_{\text {test }}$. In Appendix $\underline{\mathrm{B}}$, we examine the effect of assuming that the magnitude of $e_{\text {extrap }}$ is quadratic with the change in $d$.

In this example, we examine the benefits of including a future test by examining several cases that include future tests, one without redesign and one with redesign based on the future test by the process described in Sec. V. We will examine 10,000 possible future test outcomes $(10,000$ samples of the errors) and use 10,000 samples of the random variables. Therefore, the true probability of failure is calculated with 10,000 samples each of the response and capacity, whereas the analyst-estimated probability of failure is calculated with 10,000 samples of the capacity and 10,000 of the response by separable Monte Carlo.

\section{A. Future Test Without Redesign}

Using the 10,000 possible outcomes of the single future test, we can estimate the effectiveness of the Bayesian approach by comparing three cases. In the first case, the analyst accepts $T_{\text {calc }}$ as the best estimate of the test-article temperature. In the second case, the analyst accepts $T_{\text {meas }}$. In the third, the analyst accepts $T_{\text {Bayes }}$, where
Table 3 Comparing absolute true error when using $T_{\text {calc }}$ $T_{\text {meas }}$ and $T_{\text {Bayes }}$ as the test-article temperature

\begin{tabular}{ccc}
\hline$T$ compared & Mean error, \% & Standard deviation of error, \% \\
\hline$T_{\text {calc }}$ & 5.0 & 2.9 \\
$T_{\text {meas }}$ & 1.5 & 0.8 \\
$T_{\text {Bayes }}$ & 1.3 & 0.8 \\
\hline \hline
\end{tabular}

$T_{\text {Bayes }}$ is the temperature with the maximum likelihood in the updated distribution. Since this example simplifies the likelihood function (see Sec. IV.B.1) so that the updated distribution is uniform, we take the mean the distribution as $T_{\text {Bayes }}$. We compare the absolute error of each from the true temperature in Table $\underline{3}$.

These results show that the Bayesian approach provides the analyst with the most accurate estimate of $T_{\text {true }}$ for the test article. Accepting $T_{\text {meas }}$ results in a slightly increased error and accepting only the original $T_{\text {calc }}$ has the worst error with a mean value of $5 \%$. In 9002 of the 10,000 cases, the Bayesian approach performed as well as, or better than, just accepting the experimentally measured result; it was also better than or equal to accepting the calculated value in 8493 cases. Accepting the experimentally measured results was better than or equal to accepting the calculated value in 8493 cases as well.

In addition, we can compare the analyst-estimate probability of failure to the true probability of failure. These results are given in Table 4 . We observe that the mean true probability of failure is equal to that of the original estimated probability of failure before the test. This result is not unexpected as we did not allow redesign, thus preventing any changes in design and thus the probability of failure.

It is important to note that 8884 out of the 10,000 possible outcomes show that the true probability of failure is less than the original estimate of the probability of failure. In fact, the median true probability of failure is zero and is zero up to the 85 th percentile. A summary of the percentiles is shown in Table 5.

Based on the large number of true probability of failures that are zero, it would be expected that if redesign were implemented to restore the original estimated probability of failure, most redesigns would increase the probability of failure.

\section{B. Redesign Based on Test}

In this section, we examine the effect of deterministic and probabilistic redesign for the example. These two redesign methodologies are described in Sec. IV.D.

\section{Deterministic Redesign}

We chose deterministic redesign to occur when the ratio $T_{\text {meas }} /$ $T_{\text {calc }}\left(d_{\text {test }}, r_{\text {test }}\right)$ is greater than 1.05 (unconservative computational

Table 4 Probabilities of Failure without Redesign (using Bayesian Correction)

\begin{tabular}{lcccc}
\hline \hline Parameter & Mean & Standard deviation & Minimum & Maximum \\
\hline$p_{f, \text { true }}, \%$ & 0.121 & 0.390 & 0 & 2.00 \\
$p_{f, \text { analyst-corr }}, \%$ & 0.121 & 0.274 & 0 & 1.93 \\
\hline \hline
\end{tabular}

Table 2 Distribution of errors

\begin{tabular}{ccc}
\hline \hline Error & Distribution & Bounds \\
\hline$e_{c}$ & Uniform & $\pm 10 \%$ \\
$e_{x}$ & Uniform & $\pm 3 \%$ \\
\hline \hline
\end{tabular}

Table 5 Summary of the percentiles of the true probability of failure without redesign

\begin{tabular}{cc}
\hline \hline Percentiles, $\%$ & $p_{f, \text { true }}, \%$ \\
\hline 25 & 0 \\
50 & 0 \\
75 & 0 \\
88.8 & 0.15 \\
90 & 0.20 \\
95 & 1.10 \\
97.5 & 1.80 \\
\hline \hline
\end{tabular}


Table 6 Calibration by the correction factor approach with deterministic redesign

\begin{tabular}{lccccc}
\hline \hline Parameter & Original & Mean & Standard deviation & Minimum & Maximum \\
\hline$d_{S}, \mathrm{~mm}$ & 71.3 & 71.5 & 1.2 & 44.8 & 99.4 \\
Mass, $\mathrm{kg} / \mathrm{m}^{2}$ & 35.1 & 35.1 & 2.8 & 28.9 & 41.6 \\
$p_{f, \text { true }}, \%$ & 0.121 & $0.0007 \mathrm{ab}$ & 0.009 & 0 & 0.200 \\
\hline \hline
\end{tabular}

${ }^{a}$ Of the 10,000 possible outcomes of the future test, 4964 required redesign. Conservative cases account for 2425 of the redesigns, and unconservative cases account for 2539.

${ }^{b}$ For the true probability, $99.3 \%$ are below the mean.

Table 7 Calibration by correction factor with deterministic redesign, bounds of redesigned $d_{s}$ restricted to $\pm 10 \%$ of original $d_{s}$

\begin{tabular}{lccccc}
\hline \hline Parameter & Original & Mean & Standard Deviation & Minimum & Maximum \\
\hline$d_{S}, \mathrm{~mm}$ & 71.3 & 71.4 & 0.5 & 64.1 & 78.4 \\
mass, $\mathrm{kg} / \mathrm{m}^{2}$ & 35.1 & 35.1 & 1.2 & 33.4 & 36.7 \\
$p_{f, \text { true }}, \%$ & 0.121 & 0.0007 & 0.009 & 0 & 0.200 \\
\hline \hline
\end{tabular}

Table 8 Calibration by the Bayesian-updating approach with probability of failure based redesign $\left(p_{f, \operatorname{target}}=\mathbf{0 . 0 1 \%}\right)$

\begin{tabular}{lcccccc}
\hline \hline Restriction on redesigned $d_{s}$ & Parameter & Original & Mean & Standard deviation & Minimum & Maximum \\
\hline No bounds & $d_{S}, \mathrm{~mm}$ & 71.3 & 65.3 & 8.9 & 47.5 & 77.7 \\
& mass, $\mathrm{kg} / \mathrm{m}^{2}$ & 35.1 & 33.7 & 2.1 & 29.5 & 36.5 \\
& $p_{f, \text { true }}, \%$ & 0.121 & 0.003 ab & 0.016 & 0 & 0.100 \\
Within $\pm 10 \%$ of $d_{\text {test }}$ & $p_{f, \text { analyst-corr }}, \%$ & 0.121 & 0.007 & 0.004 & 0 & 0.015 \\
& $d_{S}, \mathrm{~mm}$ & 71.3 & 68.8 & 5.1 & 64.1 & 77.7 \\
& mass, $\mathrm{kg} / \mathrm{m}^{2}$ & 35.1 & 34.5 & 1.2 & 33.4 & 36.5 \\
& $p_{f, \text { true }}, \%$ & 0.121 & 0.003 & 0.016 & 0 & 0.100 \\
& $p_{f, \text { analyst-corr }}, \%$ & 0.121 & 0.003 & 0.005 & 0 & 0.015 \\
\hline \hline
\end{tabular}

${ }^{\mathrm{a}}$ Of the 10,000 possible outcomes of the future test, 7835 are redesigned. With the requirement of a $4.5 \%$ decrease in mass, 5126 of the 7001 conservative models $\left(p_{f \text {.analyst }}<p_{f \text { target }}\right)$ are redesigned. For unconservative designs, 2709 are redesigned.

${ }^{\mathrm{b}}$ For the true probability, $97.4 \%$ are below the mean.

model) or less than 0.95 (conservative computational model). We consider one design variable, the foam thickness $d_{s}$. This variable was chosen because it has a large impact on the bottom face sheet temperature. The results including deterministic redesign are given in Table $\underline{6}$.

These results show that the true probability of failure is greatly reduced when redesign is allowed. In addition, the standard deviation is also reduced. Since the redesign is symmetric, it does not cause much change in the average mass. The reason for this drastic reduction in probability of failure is the substantial reduction in error that allowed us to redesign all the designs that had a probability of failure above $0.121 \%$. So while the system was designed for a probability of failure of $0.121 \%$, it ended up with a mean probability of failure of $0.0007 \%$.

However, we note a large standard deviation in $d_{s}$, with the minimum and maximum values quite different from the design value of $71.3 \mathrm{~mm}$. In practice, the redesign may not be allowed to be this drastic. Therefore, we also examine the case where the bounds of the redesigned $d_{s}$ are restricted to $\pm 10 \%$ of the original nominal $d_{S}$. These results are given in Table 7 .

We observe that restricting the bounds of $d_{S}$ does not change the true probability of failure and does not cause a significant change in the average mass.

\section{Probabilistic Redesign}

The initial design does not necessarily meet the reliability requirements of the designer. It can be, for example, a candidate design in a process of design optimization. When it comes to probabilistic redesign, one may examine redesign to the mean probability without redesign or to a target probability. Here we assume the latter, and we examine cases where the target redesign probability is $p_{f \text {,target }}=0.01 \%$ with and without bounds on $d_{s}$. Here, we require redesign to occur when the estimated probability of failure is not within $\pm 50 \%$ of the target. We require that all unconservative (dangerous) designs above the 50\% threshold be redesign, but reject the redesign of overly conservative cases if its mass does not decrease by at least $4.5 \%$. Since only one design variable, the foam thickness, is considered, a decrease in mass can only result from a decrease in foam thickness, which causes an increase in temperature. The results are shown in Table 8 .

Without bounds on the redesigned $d_{s}$, we observe that the analystestimated target probability of failure is close to the target of $0.01 \%$. It is also observed that there is a significant reduction in mass $(4 \%$ reduction) and a reduction in the original mean true probability of failure from 0.121 to $0.003 \%$. The analyst is able to estimate this true probability of failure with reasonable accuracy.

When we include the bounds on $d_{s}$, the true probability of failure is unable to converge to the target probability of failure, but there is better agreement between the analyst-estimated probabilities of failure and the true value. This is due to the inclusion of the extrapolation error in the probability of failure in the redesign process. We also observe a $1.7 \%$ reduction in mean mass from the original value.

On a final note, we recognize that the large percentage of redesigns are undesirable. This percentage can be greatly reduced by less stringent redesign rules, while still having very low probabilities of failure.

\section{Conclusions}

This study presented a methodology to include the effect of a single future test followed by redesign on the probability of failure of an integrated thermal protection system. Two methods of calibration and redesign based on the test were presented. The deterministic approach, which represents current design/redesign practices, leads to a greatly reduced probability of failure after the test and redesign, a reduction that is usually not quantified. 
The probabilistic approach includes the Bayesian technique for calibrating the temperature calculation and redesign to a target probability of failure. It provides a way to more accurately estimate the true probability of failure after the test. In addition, it allows weight to be traded against performing additional tests.

Though the methodology is presented in the context of a future thermal test and redesign on the ITPS, the methodology is applicable for estimating the reliability of almost any component that will undergo a test followed by possible redesign. Given a computational model, uncertainties, errors, and redesign procedures, along with the statistical distributions, the procedure of simulating the future test result by Monte Carlo sampling, calibration, and redesign can be readily applied.

Future work includes incorporating the effect of the future test into the optimization of the ITPS. This study has brought to light many tunable parameters in the test, such as the bounds on the design variables, the target probability of failure for redesign, and the redesign criterion itself. Including these parameters into the optimization will not only optimize the design, but will optimize the test as well.

\section{Appendix A: Comparison of Bayesian Formulations}

In a rigorous formulation of the likelihood function, we would calculate the conditional probability of obtaining the measured temperature when the true temperature of the test article is $T$, as shown in Eq. (1ㅡ):

$$
l_{\text {test }}(T)= \begin{cases}\frac{1}{0.14 T} & \text { if }\left|\frac{T-T_{\text {meas }}}{T}\right| \leq 0.07 ; \\ 0 & \text { otherwise }\end{cases}
$$

In the illustrative example in Sec. IV.B.1, we simplified this formulation so that we calculated the conditional probability of obtaining $T$ given $T_{\text {meas }}$, as shown in Eq. (17). In Fig. A1, we compare the two likelihood functions and the resulting updated distribution of $f_{\text {test, } P \text { true }}^{\text {upd }}$ for the case in the example.
The figures show only a small difference in the bounds of the updated temperature distribution and the values of the PDFs. A comparison is shown in Table $\underline{\mathrm{A} 1}$.

\section{Appendix B: Extrapolation Error}

In this paper, we assumed the variation in the magnitude of the extrapolation error $e_{\text {extrap }}$ was linear with the distance of the design from the test design. The choice of this extrapolation error is very much up to the analyst, as it is a measure in the variation of the errors from the updated Bayesian estimate away from the test design. Here, we examine the effect of an assumption that the extrapolation error is quadratic, as expressed in Eq. (B1):

$$
e_{\text {extrap }}=\left(e_{\text {extrap }}\right)_{\max }\left(\frac{\left\|d-d_{\text {test }}\right\|}{\Delta d_{\text {lim }}}\right)^{2}
$$

For the example problem in Sec. VI, we estimated $e_{\text {extrap }}$ to be $2 \%$ when $d$ is changed by $\pm 10 \%$ from $d_{\text {test }}$. With the quadratic extrapolation error, this is expressed as in Eq. (B2). Because of this requirement, the magnitude of the quadratic extrapolation error is smaller for designs at a distance less than $\pm 10 \%$ away from the test design, but larger at greater distances, compared with the linear variation. We present this comparison in Fig. B1. Examining the same 10,000 possible outcomes of the future test with probabilistic redesign $\left(p_{f, \text { target }}=0.01 \%\right)$, the results in Table B1 were obtained:

$$
e_{\text {extrap }}=0.02\left(\frac{\left\|d-d_{\text {test }}\right\|}{0.1\left\|d_{\text {test }}\right\|}\right)^{2}
$$

The results show that there is improved agreement between the true and analyst-estimated probabilities of failure, as well as a slightly decreased mass and variation in the mass, with the quadratic variation in extrapolation error. Since the extrapolation error is smaller at a distance less than $\pm 10 \%$ away from the test design, the agreement between the true and analyst-estimated probabilities of failure is better with the quadratic extrapolation error. However, the agreement still suffers, due to the large magnitude of the extrapolation error at distances greater than $\pm 10 \%$.
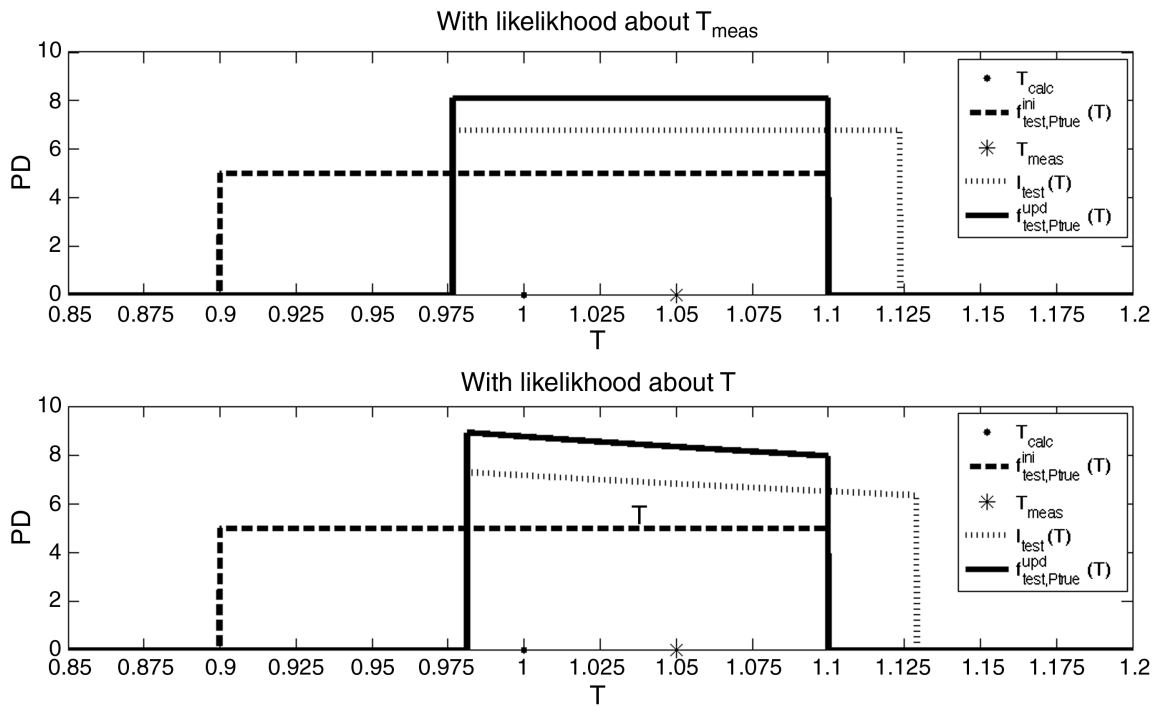

Fig. A1 Illustrative example of Bayesian updating using the likelihood about $T_{\text {meas }}$ (top) and the likelihood about $T$ (bottom).

Table A1 Comparison of $f_{\text {test }, \text { Ptrue }}^{\text {upd }}$ with different formulations of the likelihood function

\begin{tabular}{lcc}
\hline \hline Comparison & $l_{\text {test }}(T)$ about $T_{\text {meas }}$ & $l_{\text {test }}(T)$ about $T$ \\
\hline Bounds where updated distribution is nonzero & {$[0.9765,1.1]$} & {$[0.9813,1.1]$} \\
Max $f_{\text {test }, \text { Ptrue }}^{\text {upd }}$ and location & 8.1 on $[0.9765,1.1]$ & 8.9 at $T=0.9813$ \\
\hline \hline
\end{tabular}




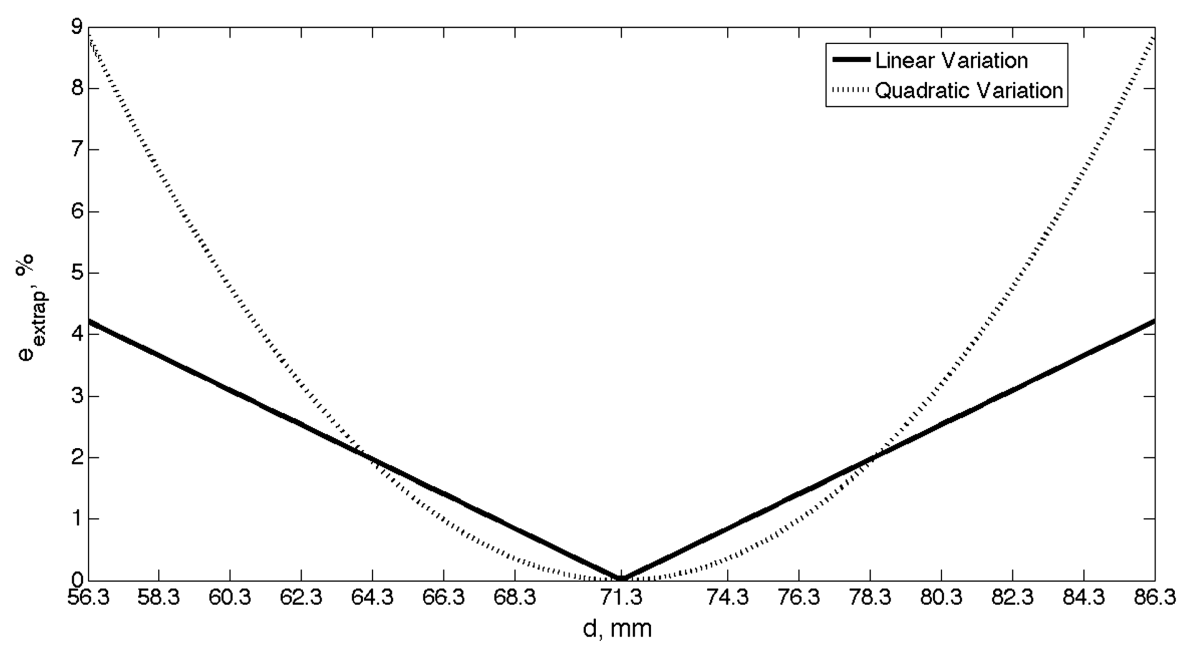

Fig. B1 Comparison of the $e_{\text {extrap }}$ with linear and quadratic variation with the distance of the design from the test design (test design is $d=71.3 \mathrm{~mm}$ ).

Table B1 Calibration by the Bayesian-updating approach with probability of failure based redesign $\left(p_{f, \text { target }}=0.01 \%\right)$, quadratic extrapolation error, and no bounds on redesign $d_{S}$

\begin{tabular}{lccccc}
\hline \hline Parameter & Original & Mean & Standard Deviation & Minimum & Maximum \\
\hline \multicolumn{5}{c}{ Linear variation in $e_{\text {extrap }}$ with $d_{s}$} \\
$d_{S}, \mathrm{~mm}$ & 71.3 & 65.3 & 8.9 & 47.5 & 77.7 \\
Mass, $\mathrm{kg} / \mathrm{m}^{2}$ & 35.1 & 33.7 & 2.1 & 29.5 & 36.5 \\
$p_{f, \text { true }}, \%$ & 0.121 & 0.003 & 0.016 & 0 & 0.100 \\
$p_{f, \text { analyst-corr }}, \%$ & 0.121 & 0.007 & 0.004 & 0 & 0.015 \\
& \multicolumn{7}{c}{ Quadratic variation in $e_{\text {extrap }}$ with $d_{s}$} \\
$d_{S}, \mathrm{~mm}$ & 71.3 & 66.4 & 7.3 & 54.4 & 77.1 \\
Mass, $\mathrm{kg} / \mathrm{m}^{2}$ & 35.1 & 33.9 & 1.7 & 31.1 & 36.4 \\
$p_{f, \text { true }}, \%$ & 0.121 & 0.004 & 0.019 & 0 & 0.100 \\
$p_{f, \text { analyst-corr }}, \%$ & 0.121 & 0.007 & 0.004 & 0 & 0.015 \\
\hline \hline
\end{tabular}

\section{Acknowledgments}

This material is based upon work supported by NASA under award no. NNX08AB40A. Any opinions, findings, and conclusions or recommendations expressed in this material are those of the author (s) and do not necessarily reflect the views of the National Aeronautics and Space Administration.

\section{References}

[1] Fujimoto, Y., Kim, S. C., Hamada, K., and Huang, F., "Inspection Planning Using Genetic Algorithm for Fatigue Deteriorating Structures," Proceedings of the International Offshore and Polar Engineering Conference, Vol. 4, International Society of Offshore and Polar Engineers, Golden, CO, 1998, pp. 99-109.

[2] Toyoda-Makino, M., "Cost-Based Optimal History Dependent Strategy for Random Fatigue Cracks Growth," Probabilistic Engineering Mechanics, Vol. 14, No. 4, Oct. 1999, pp. 339-347. doi:10.1016/S0266-8920(98)00042-3

[3] Garbatov, Y., and Soares, C., "Cost and Reliability Based Strategies for Fatigue Maintenance Planning of Floating Structures," Reliability Engineering and System Safety, Vol. 73, No. 3, 2001, pp. 293-201. doi:10.1016/S0951-8320(01)00059-X

[4] Kale, A., and Haftka, R. T., "Tradeoff of Weight and Inspection Cost in Reliability-Based Structural Optimization," Journal of Aircraft, Vol. 45, No. 1, 2008, pp. 77-85. doi:10.2514/1.21229

[5] Kale, A., Haftka, R. T., and Sankar, B. V., "Efficient Reliability-Based Design and Inspection of Panels Against Fatigue," Journal of Aircraft, Vol. 45, No. 1, 2008, pp. 86-96. doi: $10.2514 / 1.22057$

[6] Acar, E., Haftka, R. T., and Kim, N. H., "Effects of Structural Tests on
Aircraft Safety," AIAA Journal, Vol. 48, No. 10, 2010, pp. 2235-2248. doi:10.2514/1.J050202

[7] Acar, E., Haftka, R. T., Kim, N. H., and Buchi, D., "Including the Effects of Future Tests in Aircraft Structural Design," 8th World Congress for Structural and Multidisciplinary Optimization, Lisbon, Portugal, June 2009.

[8] Villanueva, D., Sharma, A., Haftka, R. T., and Sankar, B. V., "Risk Allocation by Optimization of an Integrated Thermal Protection System," 8th World Congress for Structural and Multidisciplinary Optimization, Lisbon, Portugal, June 2009.

[9] Bapanapalli, S. K., "Design of an Integrated Thermal Protection System for Future Space Vehicles," Ph.D. Dissertation, University of Florida, Gainesville, FL, 2007.

[10] Gogu, C., Haftka, R. T., Bapanapalli, S. K., and Sankar, B. V., "Dimensionality Reduction Approach for Response Surface Approximations: Application to Thermal Design," AIAA Journal, Vol. 47, No. 7, 2009, pp. 1700-1708. doi:10.2514/1.41414

[11] Oberkampf, W. L., Deland, S. M., Rutherford, B. M., Diegert, K. V., and Alvin, K. F., "Error and Uncertainty in Modeling and Simulation," Reliability Engineering and System Safety, Vol. 75, No. 3, 2002, pp. 333-357. doi:10.1016/S0951-8320(01)00120-X

[12] Smarslok, B. P., Haftka, R. T., Carraro, L., and Ginsbourger, D., "Improving Accuracy of Failure Probability Estimates with Separable Monte Carlo," International Journal of Reliability and Safety, Vol. 4, 2010, pp. 393-414. doi:10.1504/IJRS.2010.035577 